\title{
Fiber Size-Selective Stimulation using Action Potential Filtering for a Peripheral Nerve Interface: A Simulation Study
}

\author{
Adrien Rapeaux, Student Member, IEEE, Konstantin Nikolic, Member, IEEE, Ian Williams, Member, IEEE, \\ Amir Eftekhar, Member, IEEE and Timothy G Constandinou, Senior Member, IEEE
}

\begin{abstract}
Functional electrical stimulation is a powerful tool for restoration of function after nerve injury. However selectivity of stimulation remains an issue. This paper presents an alternative stimulation technique to obtain fiber size-selective stimulation of nerves using FDA-approved electrode implants. The technique was simulated for the ventral roots of Xenopus Laevis, motivated by an application in bladder control. The technique relies on applying a high frequency alternating current to filter out action potentials in larger fibers, resulting in selective stimulation of the smaller fibers. Results predict that the technique can distinguish fibers with only a $2 \mu \mathrm{m}$ difference in diameter (for nerves not exceeding $2 \mathrm{~mm}$ in diameter). The study investigates the behaviour of electrically blocked nerves in detail. Model imperfections and simplifications yielded some artefacts in the results, as well as unexpected nerve behaviour which is tentatively explained.
\end{abstract}

\section{INTRODUCTION}

Functional Electrical Stimulation (FES) is used to restore function to muscles after nervous links to the brain have been severed, as in Spinal Cord Injury (SCI). In the clinic, stimulation is carried out with FDA-approved neural electrodes such as cuff electrodes, that feature low invasiveness and reduced damage to the nerve at the cost of selectivity. Selectivity, an implant's ability to stimulate only a subpopulation of fibers in the implanted nerve, remains a major challenge due to the invasiveness and selectivity tradeoff of electrode designs. Stimulating nerves using these electrodes results in contraction of all end muscles. Efforts to increase selectivity have focused traditionally on increasing spatial selectivity of electrodes, or their ability to stimulate specific fascicles witin a nerve region. Taking an alternate approach, this paper presents a technique to selectively stimulate fibers based on their size.

In a nerve, fibers of different sizes are responsible for different functions. In this way, rather than partitioning the nerve in regions, a partitioning of function can be achieved, e.g. to avoid simultaneous stimulation of efferent and afferent fibers of different sizes. Possible applications include bladder voiding due to the different size of fibers responsible for voiding and continence [1], which would improve existing post-stimulus voiding techniques used in clinics today. For this particular technique, selective stimulation of smaller fibers in the nerve would improve the technique by avoiding contraction of the External Urethral Sphincter (EUS) [1]. However due to decreased diameter and thus surface area,

All authors are with the Department of Electrical and Electronic Engineering, Imperial College London, South Kensington Campus, London, SW7 2AZ, UK. (E-mail: \{adrien.rapeaux13, k.nikolic, i.williams10, a.eftekhar, t.constandinou\}@imperial.ac.uk)

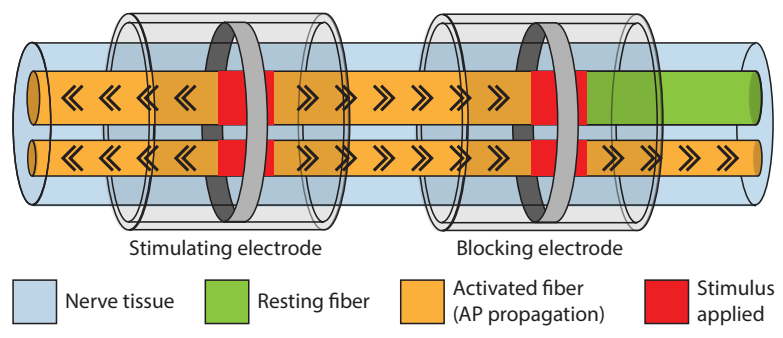

Fig. 1. Concept of selective stimulation of small nerve fibers by action potential filtering. Arrows indicate direction of propagating APs. Current return electrode is at infinity, not drawn.

smaller fibers have a higher stimulation threshold than larger fibers, and so simply adjusting stimulation amplitude will not result in selective stimulation of smaller fibers.

This paper is organised as follows: Section I introduces the work, Section II describes the concept, Section III details the methods used (tools, model, protocol), Section IV presents results, and Section V discusses/interprets the results.

\section{CONCEPT}

Rather than to modify existing stimulation techniques [2], another method is to use a selective block to prevent the large fibers from conducting. In this way action potentials (APs) inevitably generated in larger fibers never reach their destination, giving the effect of selective stimulation. This concept is shown in Fig. 1 where a proximal stimulating electrode induces APs in all the fibers of a nerve. Distally, a blocking electrode filters out APs in large fibers, resulting in selective stimulation of small fibers.

High Frequency Alternating Current (HFAC) has been used for nerve block due to its fast onset and dissipation. Kilgore and Bhadra's review of AC block [3] mention a dissipation time of 500 milliseconds. Selective HFAC blocking relies on adjusting the signal's amplitude to the block threshold of large fibers, which are blocked more easily than small fibers [4]. This is unreliable due to the varying contact quality between cuff and nerve over time. With a method using DC block [5], Kuffler has shown how a timed DC block allows filtering of APs within a nerve by separating them by propagation speed. APs propagate faster in larger fibers and are filtered out by the block, while those propagating in smaller fibers are let through when the blocking signal is shut off. The diagram he used to explain the principle of the technique is included here for clarity, Fig. 2. 


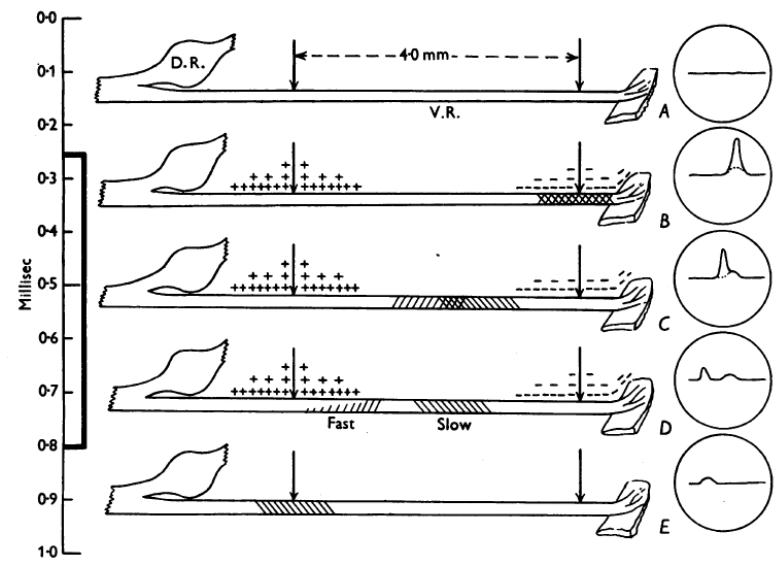

Fig. 2. Nerve preparation used by Kuffler to demonstrate a DC timing block. Time moves forward from top to bottom. The blocking and stimulating electrodes are right and left respectively, indicated by arrows. Electrode potentials are drawn as positive or negative signs. The drawings on the right show the compound action potential moving through the fiber. Adopted from [5]

Unfortunately, DC block has been shown to be damaging for nerves [6], requiring adaptation of the technique for use with HFAC. This paper investigates the use of a computational model to adapt the timing block technique to be used with HFAC .

\section{METHODS}

\section{A. Tools}

Simulations were performed using the NEURON environment [7]. The component of the extracellular electric field normal to the fiber was calculated using COMSOL Multiphysics and used within NEURON. The FrankenhaeuserHuxley (FH) [8] model was used to represent the behaviour of Xenopus Laevis neurons [9], [10]. Xenopus Laevis was used for the long ex-vivo lifetime of its nerves, the extensive literature on the animal, and its neuroanatomy: the ventral roots of Xenopus Laevis comprise two subpopulations of fibers with diameters of about $5 \mu \mathrm{m}$ and $12 \mu \mathrm{m}$ respectively, in a useful configuration for testing selective stimulation.

\section{B. Model Description}

The cuff electrode structure was selected for the model due to its low invasiveness, better chronic performance and lifetime within the body [11], and its FDA-approved status (for example in VNS therapy [12]). The model features two axons located the same distance from the blocking electrode. Only the electric fields generated by the blocking electrode are modelled using COMSOL. Nerve stimulation is modelled as an injection of current directly into both axons. A schematic for the model is shown in Fig. 3. Table I details the model parameters, obtained from [10], [13].

\section{Protocols}

The NEURON simulation features a test to judge whether selective stimulation has been achieved with the input parameters. The protocol to simulate selective stimulation described below works in reverse compared to Kuffler's work;

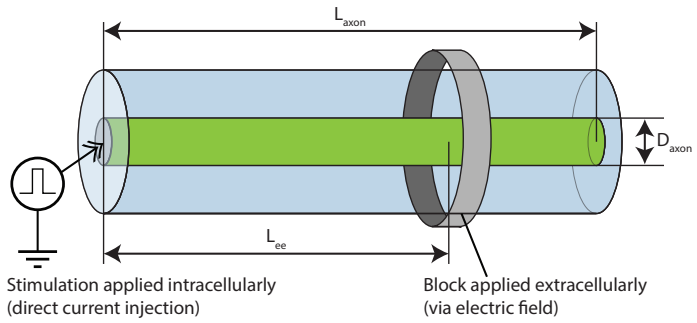

Fig. 3. Model geometry used for simulating the stimulating electrodes with intracellular wires and a spiking signal. The blocking electrodes are simulated with an extracellular electric field.

TABLE I

NERVE FIBER MODEL PARAMETERS

\begin{tabular}{|c|c|}
\hline \multicolumn{2}{|c|}{ Variable } \\
\hline Axon length & $\mathrm{L}_{\text {axon }} \mathrm{cm}$ \\
\hline Fiber diameter & $\mathrm{D}_{\text {axon }} \mu \mathrm{m}$ \\
\hline Electrode-fiber distance & $\mathrm{L}_{\text {ef }} \mu \mathrm{m}$ \\
\hline Electrode-electrode distance & $\mathrm{L}_{\text {ee }} \mu \mathrm{m}$ \\
\hline Axon Internode Length & $100 \mathrm{x} \mathrm{D}$ axon \\
\hline \multicolumn{2}{|c|}{ Constant } \\
\hline Extracellular longitudinal resistivity & $356 \Omega \cdot \mathrm{cm}$ \\
\hline Extracellular radial resistivity & $560 \Omega \cdot \mathrm{cm}$ \\
\hline Axon membrane capacitance & $2 \mu \mathrm{F} \cdot \mathrm{cm}^{-2}$ \\
\hline Myelin capacitance & $0.005 \mu \mathrm{F} \cdot \mathrm{cm}^{-2}$ \\
\hline Myelin resistivity & $29 \mathrm{M} \Omega \cdot \mathrm{cm}^{-1}$ \\
\hline Axoplasmic resistivity & $100 \Omega \cdot \mathrm{cm}$ \\
\hline Blocking electrode ring width & $1 \mathrm{~mm}$ \\
\hline Blocking electrode ring radius & $1 \mathrm{~cm}$ \\
\hline Block electrode current density & $159 \mathrm{~A} \cdot \mathrm{m}^{2}$ \\
\hline
\end{tabular}

here it is the stimulation that is timed rather than the block itself:

- The blocking signal is activated, producing an onset response as noted in [3].

- After waiting for the onset response to subside, a test pulse is sent out stimulating a single AP to be filtered.

- The timing for the stimulation pulse is calculated according to AP propagation speeds and block dissipation time using Eq. 1.

- The stimulation pulse is sent out at the calculated time and the blocking signal is removed at the set time, adjusted to ensure charge balance.

- The stimulated axons are monitored for passage of APs to determine whether selective stimulation has been achieved.

With $t_{b s o}$ the time at which the blocking signal is shut off, $t_{b d}$ the block dissipation time, and $t_{p s}$ and $t_{p f}$ the times for the slow and fast APs to reach the blocked area (directly under the blocked electrode) respectively, the timing $t_{\text {pulse }}$ of the pulse to be sent is calculated with the following equation:

$$
t_{\text {pulse }}=t_{\text {bso }}+t_{b d}-\frac{t_{p s}+t_{p f}}{2}
$$

\section{Results}

\section{A. Effect of Fiber Diameter on Block Efficiency}

The results are presented as parameter values for which a test (i.e. selective stimulation) was successful. Simulations 


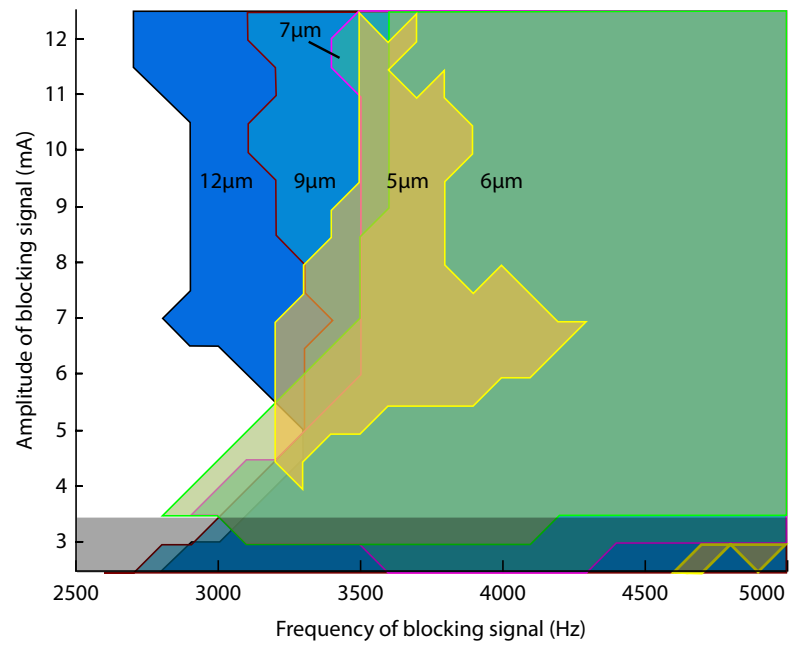

Fig. 4. Successful block parameters in signal frequency and amplitude for fibers of different diameters. Shaded portion corresponds to parameters for which there are occasional model artefacts.

were deterministic and no averaging was used. For block efficiency, the test evaluated whether total fiber block was achieved. For selective stimulation, the test evaluated whether only the small fiber conducted APs while the large fiber was blocked. For this test both fibers were at equal distance from the blocking electrode. Results present colored areas of parameters for which the test was successful, with a frequency step of $100 \mathrm{~Hz}$ and an amplitude step of $0.5 \mathrm{~mA}$.

Fig. 4 shows that small diameter fibers are harder to block than larger ones at the same distance from the electrode, since areas of parameters for which the test was successful get smaller with decreasing fiber diameter. For $5 \mu \mathrm{m}$ fibers, the lowest reliable blocking frequency was found to be about $3300 \pm 100 \mathrm{~Hz}$, with current thresholds varying from less than 2.5 to $5.5 \mathrm{~mA}$. Interestingly, the optimal blocking frequency for larger fibers is slightly higher as at $3300 \mathrm{~Hz}$ there is a range of amplitudes for which block isn't achieved for $12 \mu \mathrm{m}$ fibers.

\section{B. Effect of Electrode-Fiber Distance on Block Efficiency}

In a manner similar to the tests for the effect of fiber diameters on block efficiency, the effect of fiber to electrode distance was investigated for a $5 \mu \mathrm{m}$ fiber, with results shown Fig. 5. Similarly to the previous test, the figure shows that the farther the fiber is from the electrode, the harder it becomes to block. Beyond $1000 \mu \mathrm{m}$ of distance from the electrode, the ability of the electrode to block the nerve significantly decreases; at $1100 \mu \mathrm{m}$ of distance there are only a handful of amplitude-frequency values for the signal for which block is achieved.

\section{Effect of Interelectrode Distance on Protocol Resolution}

To determine the minimum length of the cuff to implement the protocol experimentally in an animal model, the effect of stimulating to blocking electrode distance (interelectrode distance) was investigated. The test determined if selective stimulation of a small fiber juxtaposed to a $12 \mu \mathrm{m}$ fiber was

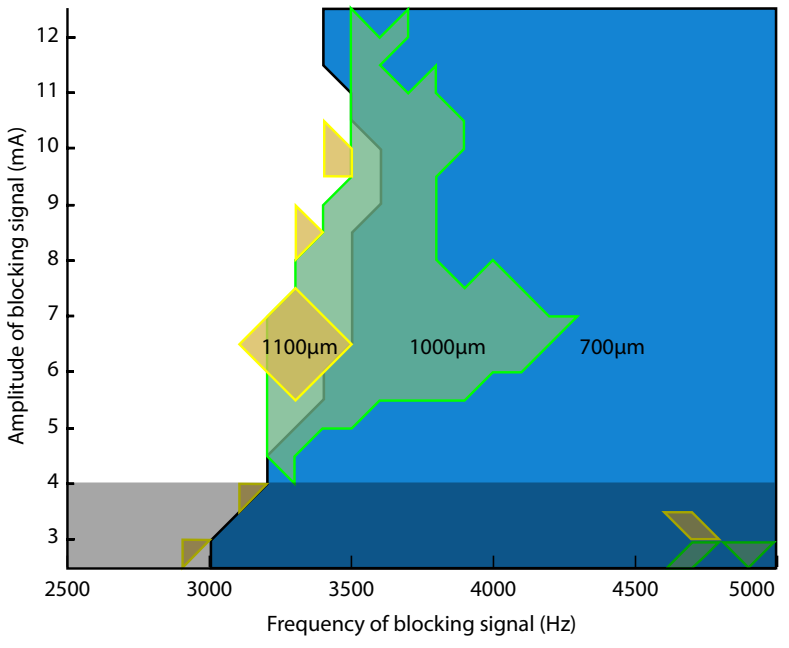

Fig. 5. Successful block parameters in signal frequency and amplitude for different electrode-fiber distances (for a $5 \mu \mathrm{m}$ diameter finer). Shaded portion corresponds to parameters for which there are occasional model artefacts.

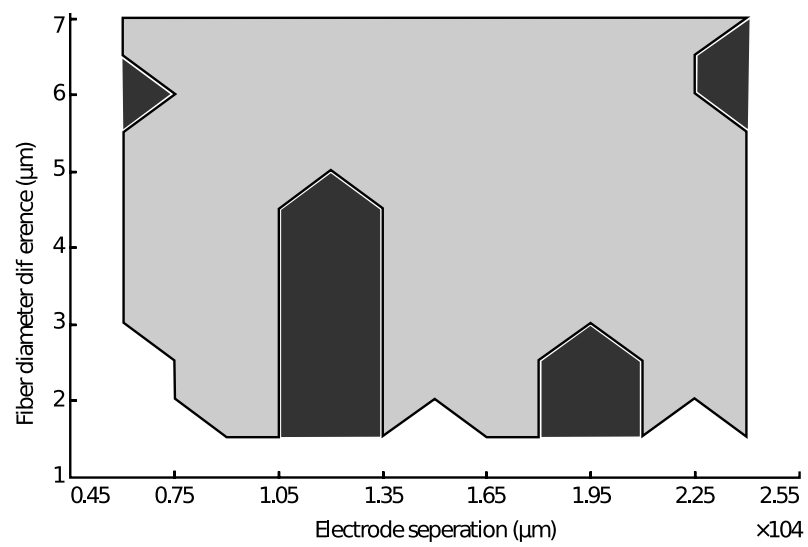

Fig. 6. Influence of inter-electrode distance on selective stimulation resolution, with a monopolar blocking electrode $1 \mathrm{~mm}$ from the fibers. Blocking signal amplitude was $5 \mathrm{~mA}$ at $3300 \mathrm{~Hz}$. The darker regions correspond to electrode-electrode distances at which the technique was much less effective, which is thought to be a model artefact.

successful, with the size difference plotted as the vertical axis in Fig. 6. The minimum distance for which selective stimulation was achieved is $6 \mathrm{~mm}$. The protocol can selectively stimulate smaller fibers with a size difference as small as $2 \mu \mathrm{m}$, determining its resolution. Model artifacts (dark blocks) cause significant reductions in the protocol's resolution at select interelectrode distances.

\section{Discussion}

The results suggest that a cuff of minimum $6 \mathrm{~mm}$ in length and a maximum diameter of about $2.2 \mathrm{~mm}$ containing two monopolar electrodes can be used for selective stimulation. The technique can be adapted to selectively stimulate larger fibers in a nerve by timing the block so that only slower action potentials are blocked, making it versatile. Smaller cuff diameters and large fiber diameter differences allow the protocol to work more effectively, though experiments using Xenopus Laevis are needed to verify the predictions in a 


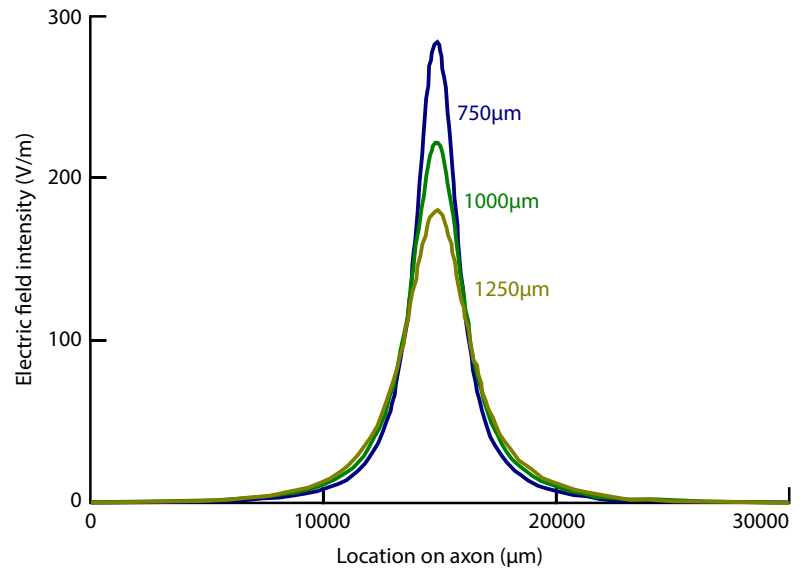

Fig. 7. Electric field (normal component) generated by a monopolar blocking electrode at several electrode-fiber distances, normalised for a $1 \mathrm{~mA}$ output from the electrode.

more realistic setting. A real nerve will feature nerve fibers at different distances from the blocking electrode. In this setting it is expected that blocking and timing parameters valid for the fiber farthest from the electrode, at the center of the nerve, will be valid for fibers closer to the surface where the electric field will be stronger and blocking more reliable as shown in previous results.

Analysis of the results for electrical block of nerve fibers shows certain unexpected behaviours that can be justified. Whereas Fig. 4 shows that the amplitude threshold for block increases with signal frequency as the nerve acts like a low-pass filter, increasing the amplitude further does not necessarily result in block of the fiber. This can be tentatively explained by observing the distribution of the component of the electric field normal to the fiber, shown in Fig. 7. At blocking threshold, shown conceptually in Fig. 8, part of the fiber's nodes of Ranvier are blocked, however nodes adjacent to the blocked region are not. These nodes can be stimulated if the electric field or signal at that location is strong enough, and so this depends on the slope of the electric field since blocking amplitude thresholds are higher than stimulation thresholds. If the slope is too low as when the fiber is far away from the electrode, no matter the signal amplitude, certain nodes adjacent to the blocked zone will be stimulated, resulting in generation of APs. Since smaller fibers have their nodes of Ranvier closer together, this explains why smaller fibers are harder to block.

Some behaviour cannot be explained however. The absence of successful blocking signal parameters beyond $4300 \mathrm{~Hz}$ for $5 \mu \mathrm{m}$ fibers $1000 \mu \mathrm{m}$ away from the blocking electrode is unexpected. It may be that the model is not realistic enough to correctly simulate the fibers at these parameters, as certain features such as paranodes are missing in the model used for this study. The presence of model artefacts that appear depending on the timestep used for the simulation also suggests the use of a real animal to verify the predictions.

It is evident that some model artifacts are present in

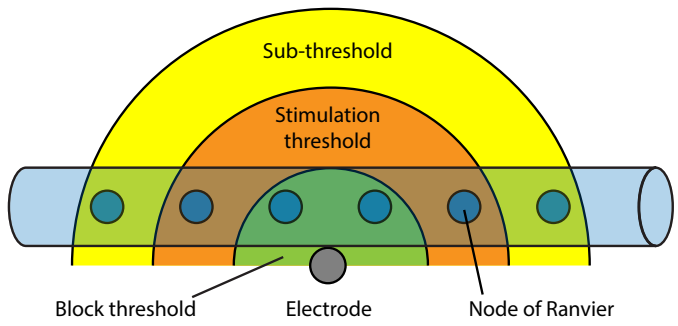

Fig. 8. Spatial variation of electric field strength into the different functional regions (sub-threshold, stimulation threshold, and blocking threshold). This explains the lack of block for certain fibers even at high signal amplitudes.

the results, causing false positives and negatives at select combinations of parameters. This is due to the fundamentally divergent nature of the model and the conservative test method used, where slight variations in parameters might cause the simulated axons to fire sporadically and trigger a negative result, or prevent such firing in the time scale of the simulations, of $25 \mathrm{~ms}$ in duration.

\section{REFERENCES}

[1] N. Rijkhoff, H. Wijkstra, P. Van Kerrebroeck, and F. Debruyne, "Urinary bladder control by electrical stimulation: review of electrical stimulation techniques in spinal cord injury," Neurology and Neurodynamics, vol. 16, no. 1, pp. 39-53, 1997.

[2] S. Luan, I. Williams, K. Nikolic, and T. G. Constandinou, "Neuromodulation: present and emerging methods," Frontiers in neuroengineering, vol. 7, 2014.

[3] K. Kilgore and N. Bhadra, "Nerve conduction block utilising highfrequency alternating current," Medical \& Biolog. Eng. and Comput., vol. 42, no. 3, pp. 394-406, 2004.

[4] M. Solomonow, "External control of the neuromuscular system," IEEE Trans. Biomed. Eng., no. 12, pp. 752-763, 1984.

[5] S. W. Kuffler and E. V. Williams, "Small-nerve junctional potentials. the distribution of small motor nerves to frog skeletal muscle, and the membrane characteristics of the fibres they innervate," J. Physiology, vol. 121 , no. 2 , pp. $289-317,1953$.

[6] J. Whitwam and C. Kidd, "The use of direct current to cause selective block of large fibres in peripheral nerves," British J. Anaesthesia, vol. 47, no. 11, pp. 1123-1134, 1975.

[7] M. L. Hines and N. T. Carnevale, "The neuron simulation environment," Neural comp., vol. 9, no. 6, pp. 1179-1209, 1997.

[8] B. Frankenhaeuser and A. Huxley, "The action potential in the myelinated nerve fibre of xenopus laevis as computed on the basis of voltage clamp data," J. Physiology, vol. 171, no. 2, pp. 302-315, 1964.

[9] M. Hines, "Xenopus myelinated neuron (frankenhaeuser, huxley 1964)," January 2002. [Online]. Available: http://senselab.med.yale.edu/ModelDB/ShowModel.asp?model=3507

[10] Z. Mou, I. F. Triantis, V. M. Woods, C. Toumazou, and K. Nikolic, "A simulation study of the combined thermoelectric extracellular stimulation of the sciatic nerve of the xenopus laevis: the localized transient heat block," IEEE Trans. Biomed. Eng., vol. 59, no. 6, pp. 1758-1769, 2012.

[11] L. Fisher, D. Tyler, J. Anderson, and R. Triolo, "Chronic stability and selectivity of four-contact spiral nerve-cuff electrodes in stimulating the human femoral nerve," J. Neural Eng., vol. 6, no. 4, p. 046010, 2009.

[12] S. C. Schachter, "Vagus nerve stimulation therapy summary: Five years after fda approval," Neurology, vol. 59, no. 6 suppl 4, pp. S15-S29, 2002

[13] J. W. Moore, R. W. Joyner, M. H. Brill, S. D. Waxman, and M. NajarJoa, "Simulations of conduction in uniform myelinated fibers. relative sensitivity to changes in nodal and internodal parameters," Biophysical J., vol. 21, no. 2, pp. 147-160, 1978. 